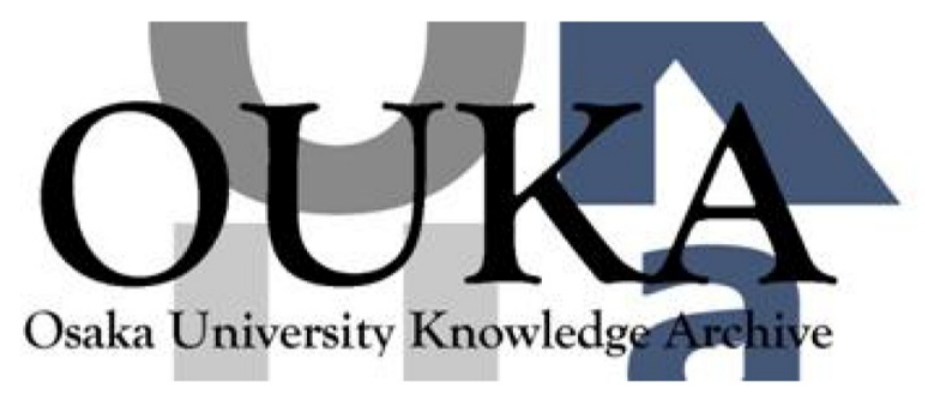

\begin{tabular}{|c|l|}
\hline Title & $\begin{array}{l}\text { Study of Thermal Smoothing by Shock Speed } \\
\text { Measurement }\end{array}$ \\
\hline Author(s) & $\begin{array}{l}\text { Kado, Masataka; Tanaka, Kazuo A.; Yamamoto, } \\
\text { Hidetoshi et al. }\end{array}$ \\
\hline Citation & プラズマ核融合学会誌. 70(8) p. 877-p. 881 \\
\hline Issue Date & $1994-08$ \\
\hline oaire:version & VoR \\
\hline URL & https://hdl. handle.net/11094/3164 \\
\hline rights & \\
\hline Note & \\
\hline
\end{tabular}

Osaka University Knowledge Archive : OUKA

https://ir. Library. osaka-u. ac. jp/

Osaka University 


\title{
Study of Thermal Smoothing by Shock Speed Measurement
}

\author{
KADO Masataka, TANAKA Kazuo A. ${ }^{1)}$, YAMAMOTO Hidetoshi, \\ VICK Doug W., TSUKAMOTO Masahiro, \\ MIYANAGA Noriaki, AZECHI Hiroshi, NISHIGUCHI Akira ${ }^{2)}$, \\ MIMA Kunioki and NAKAI Sadao \\ Institute of Laser Engineering, Osaka University, Suita, 565. \\ ${ }^{1)}$ Department of Electromagnetic Energy Engineering, Osaka University, Suita, 565. \\ ${ }^{2)}$ Osaka Institute of Technology, Asahi, Osaka 535.
}

(Received 13 January 1994/revised manuscript: received 13 June 1994)

\begin{abstract}
The smoothing of the ablation-pressure nonuniformities has been measured by the velocity profile of laser induced shock waves launched into a $\mathrm{CH}$ planar target. By changing the standoff distance (D) and the laser intensity modulation periods ( $\mathrm{L}$ ), the smoothing factor is determined and is compared to theoretical models. The dependence of the smoothing factors appears to be improved for larger values of $D / L$. This dependence is discussed, using a simple model of temperature and pressure waves. The smoothing factor obtained from experiments shows dependence of $\exp (-1.34 \times 2 \pi D / L)$ for $D / L$ less than 0.12 and $\exp (-2.34 \times 2 \pi D / L)$ for $D / L$ larger than 0.18 .
\end{abstract}

\section{Keywords:}

inertial confinement fusion, thermal smoothing, shock waves,

Intensity nonuniformities in an incident laser beam may cause corresponding variations in the ablation pressure. The variations in the ablation pressure should be less than a few percent in order for spherical targets to implode symmetrically and to be ignited in inertial confinement-fusion (ICF) experiments $[1,2]$. The uniformity requirements on the laser beam could be eased by the degree of lateral heat conduction and plasma-hydrodynamic motions occurring between the absorption and the ablation surfaces. Theoretically the scaling of the smoothing on the standoff distance (D) between the absorption and the ablation surfaces and the intensity-modulation wavelength $(\mathrm{L})$ is obtained in terms of heat conduction and plasma-fluid motions [3]. Previous experimental studies have measured the smoothing using an $x$-ray shadow graph $[4,5]$, an $x$-ray imaging $[6,7]$, and a double foil technique [8]. These experimental results were affected possibly by the plasma expansion and hydrodynamic instability. Shock velocity measurement [9] was used to infer the ablationpressure profile and to determine the smoothing with use of a square pulse. Shock speed measurement is a good way to determine the smoothing. However since several hundreds picoseconds might be necessary to achieve a steady state in this experiment, arguments assuming a steady state 
might not be valid at the time when the shock wave was launched.

In this paper we present experimental results to obtain the scaling of thermal smoothing factor on $D / L$ by temporally and spatially resolved shock speeds. A temporally Gaussian shaped laser beam was used to have time resolved smoothing factor, since the observed shock waves were the ones released at or closed to the laser intensity peak. Furthermore to obtain accurate and quantitative smoothing factors a UV-Schwarzschild microscope [10] with a high spatial resolution $(<1 \mu \mathrm{m})$ was used.

Experiments were conducted with use of a $0.53 \mu \mathrm{m}, 1 \mathrm{~ns}$ (FWHM), Gaussian shaped laser beam $\left(I_{\mathrm{L}} \sim 2-8 \times 10^{13} \mathrm{~W} / \mathrm{cm}^{2}\right)$ focused onto thin polystyrene $(\mathrm{CH})$ targets. Shown in Fig. 1 is the experimental configuration. Array of neutral density (ND) filter strips was inserted in the laser beam to obtain one dimensional periodic irradiation nonuniformity. The measured intensity ratios of the nonuniformities were ten to one or two to one on targets, thus creating intensity modulations of $4 \times$ $10^{13}$ and $4 \times 10^{12} \mathrm{~W} / \mathrm{cm}^{2}$, or $4 \times 10^{13}$ and $2 \times$ $10^{13} \mathrm{~W} / \mathrm{cm}^{2}$, for example. The modulation wavelengths $(L)$ of $50,75,100$, and $120 \mu \mathrm{m}$ were controlled by changing the focusing diameter at a

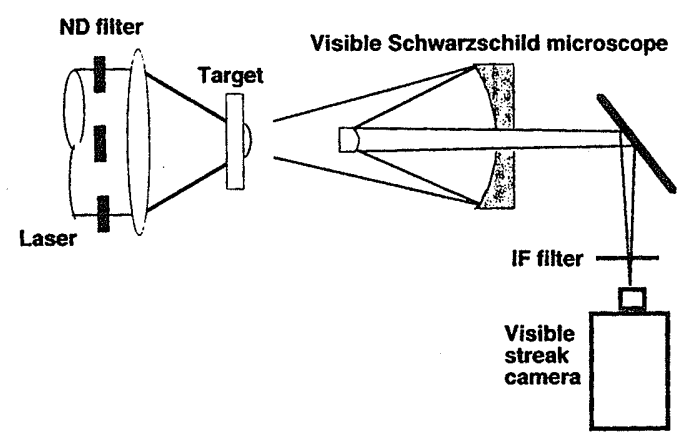

Fig. 1 Experimental configuration for temporally and spatially resolved measurement of shock waves transmitted through thin planar targets. Laser beam with an array of neutral density filter strips is focused onto a planar $\mathrm{CH}$ target, launching a shock wave in the target. Plasma emission due to shock breakout is observed through an interfarence (IF) filter with a UV-Schwarzschild microscope and is recorded with a S-20 type streak camera. constant laser intensity. The thicknesses $(T)$ of $\mathrm{CH}$ targets were changed from 10 to $25 \mu \mathrm{m}$. The combinations of $T$ (target thickness) and $L$ (perturbation wavelength) were $L=50 \mu \mathrm{m}$ for $T$ $=10$ and $15 \mu \mathrm{m}, L=100 \mu \mathrm{m}$ for $T=10,15$, and $20 \mu \mathrm{m}, L=120 \mu \mathrm{m}$ for $T=15,20$, and $25 \mu \mathrm{m}$. Varying the target thickness the transit time of a shock front was obtained with respect to the laser peak to determine the shock velocity. The measured shock fronts are the ones launched at the laser peak $[11,12]$ for the target thickness thicker than $10 \mu \mathrm{m}$, confirmed in the 1 -D fluid code HISHO [13] simulations. In this sense, the measured shock waves carry the information of the smoothing at the laser peak. Fastest shock fronts may expand in three dimensions (3D) [14] and slowest shock fronts accelerated, causing shock front oscillations. The degree of $3 \mathrm{D}$ expansion of a shock front depends on the ratio of a target thickness and a modulation wavelength. The apparent smoothing of the shock fronts could be affected by this effect which may cause an inaccurate smoothing factor estimate. In our experiment, $T / L=0.3(T=15$ and $L=50 \mu \mathrm{m}$ ) should be the one to be checked first. Since one full cycle of the oscillation may occur at $T / L$ of 2-3 [15], the slow shock front could be accelerated by $1 / 7$ cycle, resulting in $5 \%$ error for the measured shock speeds. This effect is also experimentally verified separately. The $3 \mathrm{D}$ effect on the smoothing factor was measured to be less than $10 \%$ for the target thickness thinner than $25 \mu \mathrm{m}$.

When the shock wave breaks out the rear of the $\mathrm{CH}$ planar target, the shock heated plasma $\left(T_{\mathrm{e}}\right.$ $<50 \mathrm{eV}$ ) produces a close-to-black body radiation $[12,16]$. The arrival time of this shock emission was measured with a Schwarzschild-type microscope coupled with an S-20 streak camera. The observed wavelength was from 380 to $480 \mathrm{~nm}$ using an interference filter.

Figures $2(\mathrm{a})$ and (b) are the experimental results without or with the ND filter array. Figure 2 (a) shows a uniform shock front profile in the 20 $\mu \mathrm{m}$ target thickness and the shock speed is $3 \times$ $10^{6} \mathrm{~cm} / \mathrm{s}$ at the laser intensity of $4 \times 10^{13} \mathrm{~W} / \mathrm{cm}^{2}$. Figure 2(b) shows a modulated shock-front profile for the $100-\mu \mathrm{m}$ modulation wavelength with the same target thickness as Fig. 2 (a) The speeds of 
(a) without ND filter
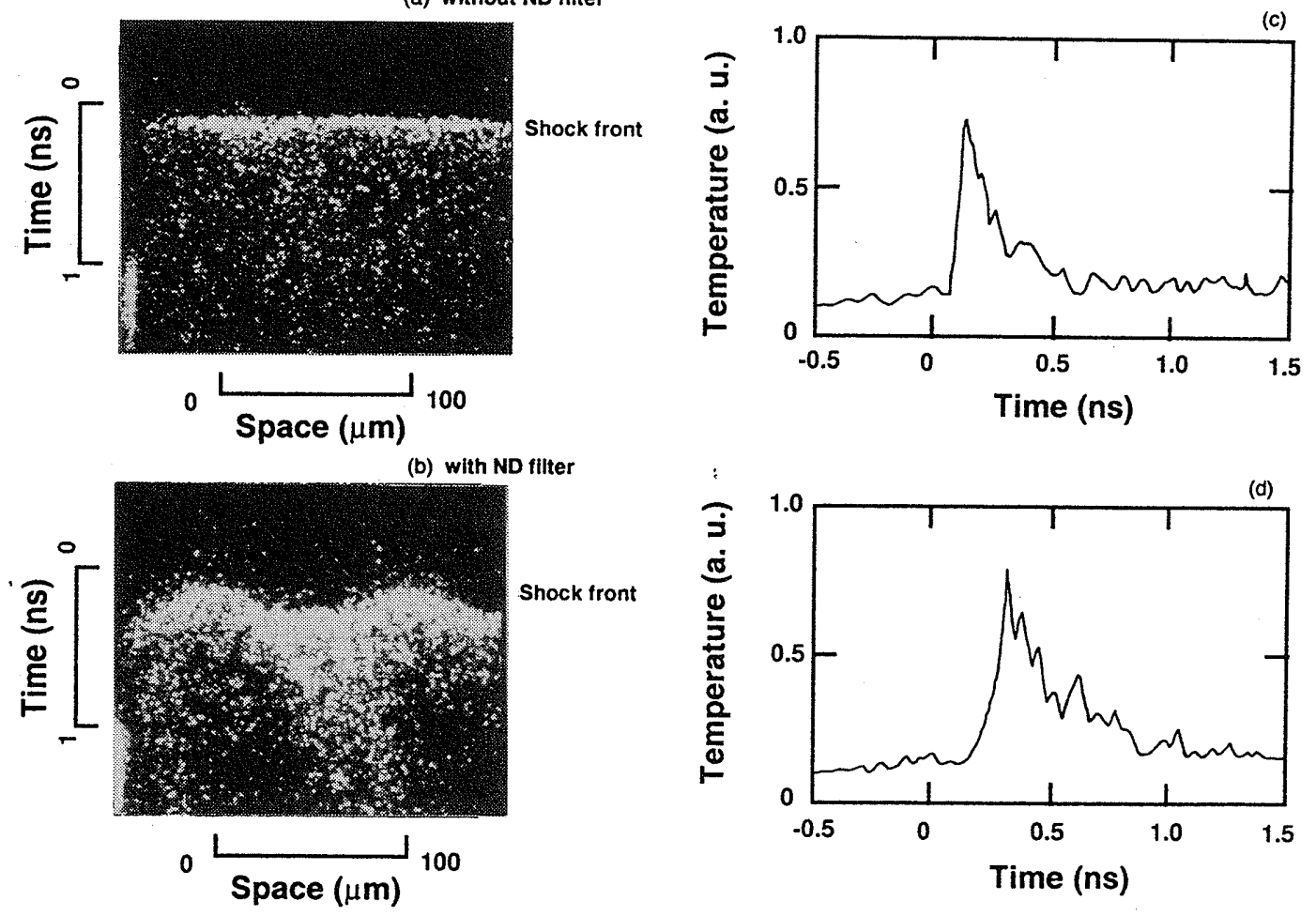

Fig. 2 Streak-camera record of the rear surface emission of a $\mathrm{CH}$ target (a) without and (b) with laser nonuniformities. The sinusoidal shock fronts are observed with the nonuniform laser irradiation. At the lower left hand corners, time fiducial marks are shown. The modulation wavelength is $100 \mu \mathrm{m}$ in Fig. 2(a). Figures $2(\mathrm{c})$ and (d) are the temporal profiles of the shock fronts emission for the fast and slow shocks. This intensity corresponds to the shock front temperature under the black body condition. Time $=0$ shows the laser peak.

the fast and slow shock fronts were $3.0 \times 10^{6} \mathrm{~cm} /$ $\mathrm{sec}$ and $2.5 \times 10^{6} \mathrm{~cm} / \mathrm{s}$ and correspond to the highest $\left(I_{\mathrm{L}}=4 \times 10^{13} \mathrm{~W} / \mathrm{cm}^{2}\right)$ and lowest $\left(I_{\mathrm{L}}=4\right.$ $\times 10^{12} \mathrm{~W} / \mathrm{cm}^{2}$ ) intensities. Figures $2(\mathrm{c})$ and $(\mathrm{d})$ are the time histories of the emission intensity of the fast and slow shock fronts. Time $=0$ indicates the laser peak. These intensities correspond to shock front temperature if the emission is a black body. Both shock fronts of Fig. 2 (c) and (d) showed a fast rise time $(\sim 100 \mathrm{ps})$, typical for the strong shock wave. The smoothing factor is defined as the ratio of perturbed ablation pressures with and without the thermal smoothing. Here the smoothing factor $\Gamma$ is calculated using the dependence [17] of $P_{\mathrm{a}} \propto$ $I_{\mathrm{a}}{ }^{2 / 3}$ and $P_{\mathrm{a}} \propto V^{2}$ as,

$$
\begin{aligned}
& \Gamma=\frac{1}{2} \frac{\delta P_{\mathrm{a}}}{\bar{P}_{\mathrm{a}}} \frac{I_{\mathrm{a}, \max .}^{2 / 3}+I_{\mathrm{a}, \min }^{2 / 3}}{I_{\mathrm{a}, \max .}^{2 / 3}-I_{\mathrm{a}, \min .}^{2 / 3}} \\
& =\frac{\delta V}{\bar{V}} \frac{I_{\mathrm{a} \text {, } 3 a x}^{2 / 3}+I_{\mathrm{a}, \min }^{2 / 3}}{I_{\mathrm{a}, \text { max. }}^{2 / 3}-I_{\mathrm{a}, \text { min. }}^{2 / 3}}, \\
& \delta P_{\mathrm{a}}=P_{\mathrm{a}, \max .}-P_{\mathrm{a}, \min .}, \quad \delta V=V_{\text {max. }}-V_{\text {min. }}
\end{aligned}
$$

were $\bar{P}_{\text {a }}$ and $\bar{V}$ are the average ablation pressure and the shock velocity. Subscripts, max. and min, indicate the maximum and minimum values of each quantity.

The smoothing factor $\Gamma$ is usually scaled with $\exp (-\alpha k D)$, where $k$ is the wave number of the spatial perturbations of an incident laser beam, $D$ the standoff distance, $\alpha$ the fitting factor. Theore- 


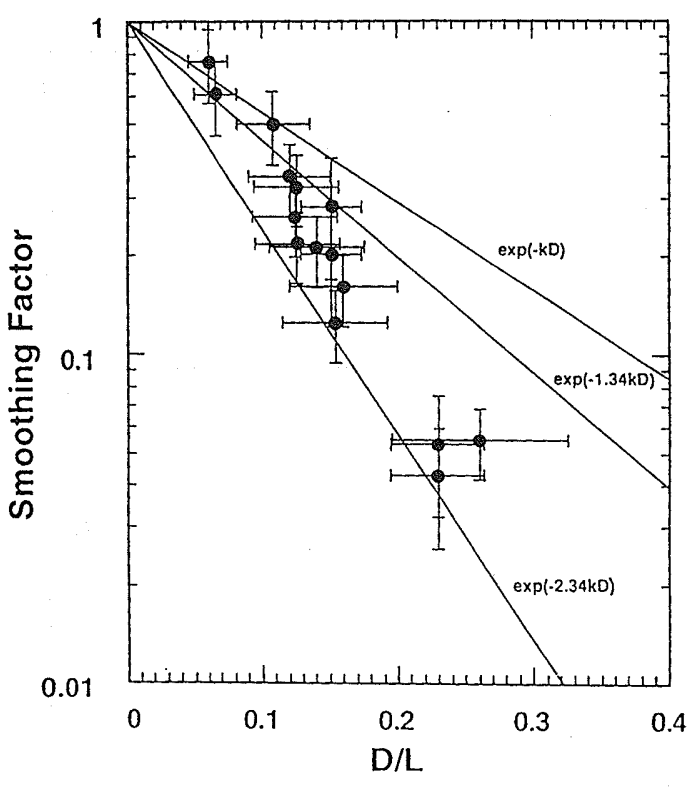

Fig. $3 \mathrm{D} / L$ dependence of the obtained smoothing factor. The closed circles show the experimental results. The smoothing factor shows an exponential decrease as $D / L$ increased. Also plotted are lines predicted by the cloudyday model, the sound-wave model, and the model independently combining the sound-wave and the cloudy-day models. The $D / L$ dependence of the smoothing factor agreed with the simple cloudy-day model $(\Gamma=\exp (-k D))$ or the sound wave model $(\Gamma=\exp (-1.34 k D))$ for $D / L$ smaller than 0.12 . For $D / L$ larger than 0.12 the smoothing factor is improved rapidly and approaches to $\Gamma=\exp (-2.34 k D)$ fitting.

tically $\alpha$ is expected to be 1 for the cloudy-day model and 1.34 for the sound-wave model [3]. Here we use the distance between the ablation and the cut off surface as $D$ for simplicity. We estimate the possible difference of the $D$ from the separation distance between the ablation and the absorption surface could be $20 \%$ at most.

Shown in Fig. 3 is the $D / L$ dependence of the obtained smoothing factors, where $D$ is obtained from HISHO. Here an absorption rate of $80 \%$ at the maximum laser intensity and $100 \%$ at the minimum laser intensity are assumed from HISHO. The error of the absorption rate is expected to be less than $10 \%$. The standoff distance from HISHO agrees within $10 \%$ with the one in a steady state by Manheimer et al. [3] and Fabbro et al. [18]. D, for example, is $12 \mu \mathrm{m}$ in HISHO and $11 \mu \mathrm{m}$ in the steady state model at the laser intensity of $4 \times 10^{13} \mathrm{~W} / \mathrm{cm}^{2}$ under our experimental conditions. This coincidence indicates that the standoff distance at the laser peak with a Gaussian pulse may be regarded as the one in a close-to steady state at that laser intensity. $D / L$ was changed by varying the laser intensity and the modulation wavelength.

The smoothing factor shows an exponential decrease as $D / L$ increases in Fig. 3. Also plotted are lines predicted by the cloudy-day model $(\Gamma=$ $\exp (-k D))$, the sound-wave model $(\Gamma=\exp (-$ $1.34 k D)$ ), and the model independently combining the cloudy-day model and the sound-wave model $(\Gamma$ $=\exp (-2.34 k D))$. The $D / L$ dependence of the smoothing factor agrees with the simple cloudy-day model or the sound wave model for $D / L$ smaller than 0.1 . For $D / L$ larger than 0.1 the smoothing factor is improved rapidly and approaches to $\Gamma=$ $\exp (-2.34 k D)$ fitting.

Thermal electrons and ions transport absorbed laser energy laterally as thermal and sound (pressure) waves, contributing to thermal smoothing. Experimental results showed that $D / L$ dependence of the smoothing factor is close to the steady state model taking account of thermal and sound waves for large $D / L$. The ablation in a $1 \mathrm{D}$ steady state may be defined as $\tau_{\mathrm{L}} v_{\text {sound }} / D>1$, where the $\tau_{\mathrm{L}}$ is the laser pulse width and $v_{\text {sound }}$ the ion sound velocity. In our experimental condition, for example $\tau_{\mathrm{L}}=1 \mathrm{~ns}, v_{\text {sound }}=1 \times 10^{7} \mathrm{~cm} / \mathrm{s}$, and $D$ $<30 \mu \mathrm{m}$, this criterion is always satisfied. Threshold speeds of the thermal and sound waves for a 2D steady state ablation could come from the time limitation within which that the thermal and pressure waves of the maximum intensity area should flow laterally into the minimum intensity area by the time of the laser peak. The ablation in the $2 \mathrm{D}$ steady state is defined as $\tau_{\mathbf{L}} v_{\text {thermal }} / L>1$ and $\tau_{\mathrm{L}} v_{\text {sound }} / L>1$, where $v_{\text {thermal }}$ is the thermal wave speed. The thermal wave [19] and the sound wave speeds are expressed as the function of $T_{\mathrm{e}}$ and $L$ as,

$$
v_{\text {thermal }}=\frac{K \nabla k_{\mathrm{B}} T_{\mathrm{e}}}{\rho C_{\mathrm{p}} T_{\mathrm{e}}}=4.46 \times 10^{-2} \frac{T_{\mathrm{e}}^{5 / 2}}{L},
$$




$$
v_{\text {sound }}=\left[\frac{\gamma Z k_{\mathrm{B}} T_{\mathrm{e}}}{m_{\mathrm{i}}}\right]^{1 / 2}=9.10 \times 10^{5} T_{\mathrm{e}}^{1 / 2},
$$

where $K$ is the thermal conductivity, $k_{\mathrm{B}}$ the Boltzman constant, $T_{\mathrm{e}}$ the electron temperature, $\rho$ the mass density, $C_{\mathrm{p}}$ the specific heat constant, $\gamma$ the adiabatic constant, $Z$ the atomic number, and $m_{\mathrm{i}}$ the ion mass. Using the experimental values for $\tau_{\mathrm{L}}$ and $L$, the thershold $D$ or the threshold $D / L$ of $2 \mathrm{D}$ steady state ablation is determined. Here the steady state standoff distance $D=0.16 K T_{\mathrm{e}}^{2} / \rho$ [3] is used. The threshold $D / L$ for the sound wave is 0.03. The threshold $D / L$ for the thermal wave varies from 0.12 to 0.18 , depending on the experimental conditions. The sound wave always satisfies the threshold condition at these $D / L$ values. Here the thermal conductivity is expressed as $K=\left(3.7 \times 10^{-33} A^{7 / 2}\right) /\left\{(1+Z)^{7 / 2} Z_{\text {eff }} \ln \Lambda\right\}$. The temperature gradient, $\nabla T_{\mathrm{e}}$, could be defined roughly as $2 T_{\mathrm{e}} / L$. A indicates the atomic number, $Z_{\text {eff }}$ the effective $Z$ for collisional process, and $\ln A$ the Coulomb logarithm. The parameters for these calculations are 3.5 for both $Z_{\text {eff }}$ and $Z, 7$ for $A, 50$ to $120 \mu \mathrm{m}$ for $L, 1.1 \mathrm{~g} / \mathrm{cm}^{3}$ for $\rho$, and 5 to 8 for $\ln \Lambda$. Since the criterion of the 2D steady state is satisfied for both waves in $D / L$ larger than 0.18 , the smoothing factor is expressed as the combination of thermal (cloudy-day) and sound wave models. In this case the smoothing factor is close to the product of thermal and sound wave models exp $(-2.34 k D)$. Next we discuss transient (not steady state) region $(D / L<0.12)$. The transient region is distingulshed into two parts; (1) where both thermal and sound waves do not satisfy the 2D steady state conditions $1>\tau_{\mathrm{L}} v_{\text {sound }} / L>\tau_{\mathrm{L}} v_{\text {thermal }} / L$ and (2) where only the sound wave satisfies the $2 \mathrm{D}$ condition $\tau_{\mathrm{L}} v_{\text {sound }} / L>1>\tau_{\mathrm{L}} v_{\text {thermal }} / L$. In the region (1) $D / L<0.03$, there is no smoothing and the smoothing factor $\Gamma$ becomes 1 . In the region (2) $0.03<D / L<0.12$, only sound wave may contribute to thermal smoothing with the smoothing factor $\Gamma=\exp (-1.34 k D)$. The fitting of the sound wave for $D / L$ smaller than 0.12 and the thermal and sound waves for $D / L$ larger than 0.12 all seem to agree quite well with the observation as shown in Fig. 3.

In summary, the $D / L$ scaling of the smoothing factor was obtained experimentally by the shock velocity measurement with high spatial and temporal resolutions. The smoothing factor $(\Gamma)$ appears to be improved at $D / L$ larger than 0.12 . Then we evaluate the energy-carrier speeds of the sound and thermal waves in terms of $D / L$. This model shows that when the $D / L$ is less than 0.12 , the perturbations are carried and smoothed mainly by the sound wave. Then the smoothing factor is represented as $\exp (-1.34 k D)$. As the $D / L$ is larger than 0.18 , the thermal wave starts contributing to thermal smoothing factor, resulting in $\Gamma=\exp (-2.34 k D)$.

\section{References}

[1] S. Bodner, Nuclear Fusion 27, 505 (1987).

[2] J. H. Gardner and S. E. Bodner, Phys. Rev. Lett. 47, 1137 (1981).

[3] W. M. Manheimer, D. G. Colombant and J. H. Gardner, Phys. Fluids 25, 1644 (1982).

[4] J. L. Bocher, et al., Phys. Rev. Lett. 52, 823 (1984).

[5] A. J. Cole et al., J. Phys. D15, 1689 (1982).

[6] W. C. Mead et al., Phys. Fluids 27, 1301 (1984).

[ 7 ] I. Matsushima et al., J. Appl. Phys. 58, 1674 (1985).

[8] S. P. Obenschain et al., Phys. Rev. Lett. 46, 1402 (1981).

[9] C. L. Shepard and P. M. Campbell, Phys. Rev. A39, 1344 (1989).

[10] M. Kado et al., Optics Letters 16, 109 (1991).

[11] D. Salzmann et al., Phys. Rev. A28, 1738 (1983).

[12] R. Kodama et al., Phys. Fluids B3, 735 (1991).

[13] K. A. Tanaka et al., in ECLIM Proceeding 21, Laser Interaction with Matter, eds. H. Fiedrowicz et al. (Warsaw, 1991), pp.201-204.

[14] F. Cottet et al., J. Appl. Phys. 64, 4474 (1988).

[15] David H. Muruno, Phys. Fluids B1, 134 (1988).

[16] A. Yamauchi et al., Appl. Phys. Lett. 52, 786 (1988); R. J. Trainer and Y. T. Lee, Phys. Fluids 25, 1898 (1982); F. Cottet and J. P. Romain, Phys. Rev. Lett. 52, 1884 (1984).

[17] C. E. Max, C. F. Mckee, and W. C. Mead, Phys. Fluids 23, 1620 (1980); C. E. Max, C. F. Mckee, and W. C. Mead, Phys. Rev. Lett. 45, 28 (1980).

[18] R. Fabbro, C. Max, and E. Fabre, Phys. Fluids 28, 1463 (1985)

[19] Ya. B. Zeldovichi and Yu. P. Raizer, Physics of Shock Waves and High Temperature Hydrodynamic Phenomena (Academic, New York, 1966), Vol.II, p.652. 